\title{
SCIENTIFIC REP RTS OPEN Author Correction: The Oncogenic Role of COL23A1 in Clear Cell Renal Cell Carcinoma
}

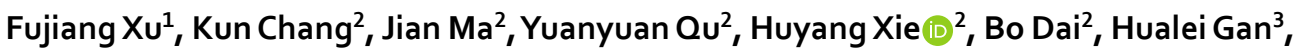 Hailiang Zhang ${ }^{2}$, Guohai Shi ${ }^{2}$, Yao Zhu ${ }^{2}$, Yiping Zhu ${ }^{2}$, Yijun Shen ${ }^{2}$ \& Dingwei $\mathrm{Ye}^{4}$}

Correction to: Scientific Reports https://doi.org/10.1038/s41598-017-10134-2, published online 29 August 2017

In the original version of this Article there were errors with the affiliations which were incorrectly listed as 'Department of Urology, Fudan University Shanghai Cancer Center, Shanghai, 200032, China', 'Institutes of Biomedical Sciences, Fudan University, Shanghai, 200032, China, 'Department of Oncology, Shanghai Medical College, Fudan University, Shanghai, 200032, China' and 'Department of Pathology, Fudan University Shanghai Cancer Center, Shanghai, 200032, China'.

The correct affiliations are listed below.

Affiliation 1:

Department of Urology, Fudan University Shanghai Cancer Center, Institutes of Biomedical Sciences, Fudan University, Shanghai, 200032, China.

Affiliation 2:

Department of Urology, Fudan University Shanghai Cancer Center, Department of Oncology, Shanghai Medical College, Fudan University, Shanghai, 200032, China.

Affiliation 3:

Department of Pathology, Fudan University Shanghai Cancer Center, Department of Oncology, Shanghai Medical College, Fudan University, Shanghai, 200032, China.

Affiliation 4:

Department of Urology, Fudan University Shanghai Cancer Center, Institutes of Biomedical Sciences, Department of Oncology, Shanghai Medical College, Fudan University, Shanghai, 200032, China.

These errors have now been corrected in the PDF and HTML versions of the Article, and in the accompanying Supplementary Information file. 
(i) Open Access This article is licensed under a Creative Commons Attribution 4.0 International License, which permits use, sharing, adaptation, distribution and reproduction in any medium or format, as long as you give appropriate credit to the original author(s) and the source, provide a link to the Creative Commons license, and indicate if changes were made. The images or other third party material in this article are included in the article's Creative Commons license, unless indicated otherwise in a credit line to the material. If material is not included in the article's Creative Commons license and your intended use is not permitted by statutory regulation or exceeds the permitted use, you will need to obtain permission directly from the copyright holder. To view a copy of this license, visit http://creativecommons.org/licenses/by/4.0/.

(C) The Author(s) 2018 